\title{
Time-dependent 3D modulation of Jovian electrons
}

\section{Comparison with Ulysses/KET observations}

\author{
D. Lange, H. Fichtner, and R. Kissmann
}

Institut für Theoretische Physik IV, Weltraum- und Astrophysik, Ruhr-Universität, Bochum, Germany

e-mail: dennie@tp4.rub.de

Received 17 August 2005 / Accepted 27 October 2005

\section{ABSTRACT}

We report on the modelling of the time-dependent transport of a few $\mathrm{MeV}$ electrons in the heliosphere. These low-energetic electrons, observed with the Kiel Electron Telescope (KET) on board the Ulysses spacecraft, are for the first time simulated over a complete solar activity cycle on the basis of a time-dependent three-dimensional modulation model. It is demonstrated that the solar activity has an effect especially at high heliographic latitudes being observed by Ulysses. The findings of the Ulysses mission lasting almost 15 years now provide a substantial data base for this study. Both galactic cosmic rays and the Jovian electron source are considered for the simulation of the electron fluxes within the heliosphere. Originating from these simulations two models for the time-dependent solar wind expansion are suggested, formulated and tested. Both characterise the varying velocity field of the solar wind over a solar cycle. A time-dependent anisotropic diffusion tensor was also tested in order to figure out from KET observations the variation of the release rate of low-energetic electrons from the Jovian magnetosphere.

Key words. Sun: solar wind

\section{Introduction}

The NASA/ESA spacecraft Ulysses, which reached high heliographic latitudes for the very first time, was launched on 6 October 1990. To date, lasting now almost 15 years, Ulysses orbits the Sun for the third time. The first orbit, in the years from 1990 to 1998 , coincided with solar minimum and the second orbit (1998-2004) with solar maximum conditions. Accordingly, Ulysses has observed one complete 11-year solar activity cycle. This cycle has an important influence on the transport processes of charged particles in the heliosphere, especially on the transport of Jovian electrons as is demonstrated in this paper. Jovian electrons are the dominant low-energy electron component at low to mid heliographic latitudes for heliocentric distances less than about 20 AU (Ferreira 2001a,b). With the Ulysses/KET data, which span more than ten years, it is now possible to compare transport model results with observations in great detail, and that allows an explicit consideration of the time dependence of the electron fluxes. In addition, the Ulysses/SWOOPS measurements give a detailed picture of the latitudinal solar wind structure and its variations with the solar cycle.

\section{Modulation model}

The basic concept of our approach to a time-dependent threedimensional modelling of electron flux modulation in the heliosphere is a momentum integration of Parker's transport equation (Parker 1965). For this we use the following definition of the electron pressure (see Fichtner et al. 1996):

$P_{\mathrm{e}}(\boldsymbol{r}, t)=\frac{4 \pi}{3} \int_{0}^{\infty} f(\boldsymbol{r}, p, t) p w p^{2} \mathrm{~d} p$

where $w$ is the particle speed. In view of negligible particle drifts at the energy range of interest, the transport equation then takes the form:

$\frac{\partial P_{\mathrm{e}}}{\partial t}=\nabla \cdot\left(\kappa \nabla P_{\mathrm{e}}\right)-\boldsymbol{u}_{\mathrm{sw}} \cdot \nabla P_{\mathrm{e}}-\frac{4}{3}\left(\nabla \cdot \boldsymbol{u}_{\mathrm{sw}}\right) P_{\mathrm{e}}$,

where $\boldsymbol{u}_{\mathrm{sw}}$ is the solar wind velocity and $\kappa$ has to be understood as the momentum-averaged diffusion tensor, that is $\kappa(\boldsymbol{r}, t) \equiv\langle\kappa(\boldsymbol{r}, p, t)\rangle=\kappa(\boldsymbol{r}, \tilde{p}, t)$, with a suitable $\tilde{p}=$ const. In the following the diffusion tensor is evaluated at a momentum $\tilde{p}$ corresponding to an energy of $7 \mathrm{MeV}$. The resulting $P_{\mathrm{e}}(\boldsymbol{r}, t)$ exhibits very similar space and time variations as the phase space distribution $f(\boldsymbol{r}, \tilde{p}, t)$ in the (original) Parker equation. The four-dimensional integrated Parker transport Eq. (2) is the basis for our electron flux simulations in the heliosphere. Consequently, the two unknown functions are the solar wind velocity and the diffusion tensor. The real unknown, however, is just the diffusion tensor, because the findings of the Ulysses mission give a detailed picture of the structure of the solar wind velocity field. These measurements of SWOOPS onboard the Ulysses spacecraft are discussed in the next chapter. 


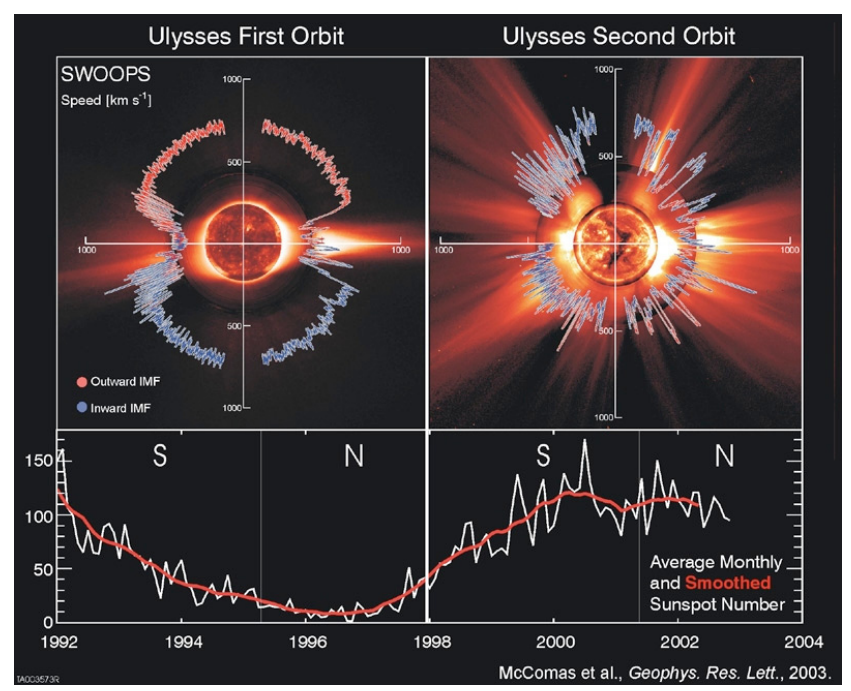

Fig. 1. Measurement of the Ulysses/SWOOPS instrument. The left panel shows measurements during the solar minimum phase (1992-1998), while the right panel during the solar maximum phase (1998-2004). The two bottom panels show the average monthly and smoothed sunspot number. Source: McComas et al. (2003).

\section{The solar wind measurements of Ulysses}

Ulysses is the first spacecraft to undertake measurements far from the ecliptic plane and over the polar regions of the Sun. One of many scientific instruments onboard Ulysses is SWOOPS (Solar Wind Observations Over the Poles of the Sun). This instrument is measuring solar wind velocity. Figure 1 shows the latitudinal dependence of the solar wind speed measured by SWOOPS for the period from 1992 to 2004. The top left panel of Fig. 1 shows the measurements during the first orbit of Ulysses (1992-1998), the top right panel during the second orbit (1998-2004). The two bottom panels display the average monthly (and smoothed) sunspot number. Evidently, the first orbit fell into the phase of solar minimum and the second orbit into solar maximum. In the top left panel (solar minimum conditions) fast solar wind was observed at high latitudes, whereas in the equatorial regions of the Sun slow solar wind speed is dominant. The region of this slow solar wind with speeds lower then $400 \mathrm{~km} \mathrm{~s}^{-1}$ is called the streamer belt, which is also associated with high plasma densities and closed magnetic field lines.

These contrast with the coronal holes at high latitudes, where densities are low and field lines are open. It has long been established that coronal holes are sources of high-speed solar wind. At solar minimum two principal coronal holes are located near the solar poles and extend towards the solar equator. Because of this, high speed solar wind (higher then $400 \mathrm{~km} \mathrm{~s}^{-1}$ up to $800 \mathrm{~km} \mathrm{~s}^{-1}$ ) was observed by Ulysses at higher latitudes. The boundary, which characterises the transition from low to high solar wind speed, is specified by the extent of the streamer belt with respect to the solar equator. The angle of inclination is about $20^{\circ}$ at solar minimum conditions. Some authors, e.g. Ferreira (2002), use $35^{\circ}$, but a closer examination of the SWOOPS data (Fig. 1) reveals an inclination angle of $20^{\circ}$ (see also Moeketsi et al. 2005). As shown in the right upper panel of Fig. 1, at solar maximum the latitudinal solar wind structure differs markedly from solar minimum conditions. During Ulyssest's second orbit fast wind is absent and now a highly variable slow solar wind extends over all latitudes with an average speed of $400 \mathrm{~km} \mathrm{~s}^{-1}$. The conditions of the streamer belt dominate all over the Sun. The opening of the streamer belt varies over the course of a solar cycle such that it is lowest near solar minimum and highest near solar maximum, when the polarity of the solar magnetic field changes sign. These strong variations are correlated with coronal holes, which are statistically distributed over the Sun at solar maximum. A model for the latitudinal variation of the velocity field of the solar wind with solar cycle is discussed in this paper. In the next chapter two models are suggested and described in detail.

\section{Modeling the varying velocity field of the solar wind}

To model the solar wind velocity in the modulation model it is assumed that

$\boldsymbol{u}_{\mathrm{sw}}(r, \Theta)=v_{0} \cdot v_{\mathrm{r}} \cdot f_{\Theta} \cdot \boldsymbol{e}_{\mathrm{r}}$,

where $r$ is the radial distance, $\Theta$ the polar angle and $\boldsymbol{e}_{\mathrm{r}}$ the unit vector in the radial direction. Any non-radial propagation is neglected. $v_{0}$ denotes the slow wind of $400 \mathrm{~km} \mathrm{~s}^{-1}$. The radial dependence $v_{\mathrm{r}}(r)$ is given as (Ferreira 2002):

$v_{\mathrm{r}}=1-\exp \left(\frac{40}{3}\left(\frac{r_{\mathrm{s}}-r}{r_{0}}\right)\right)$

with $r$ and $r_{\mathrm{s}}$ (radius of the Sun), given in AU, with $r_{0}=$ 1 AU. From this form it follows that the solar wind is accelerated about close to the Sun and reaches a constant speed at a heliocentric distance of $0.3 \mathrm{AU}$. For the latitudinal dependence $f_{\Theta}(\Theta)$ during solar minimum conditions we use the form (Ferreira 2002):

$$
\begin{gathered}
v_{0} \cdot f_{\Theta}=v_{0} \cdot\left\{\begin{array}{l}
1.5-0.5 \tanh \left(8\left(\Theta-\frac{\pi}{2}+\Theta_{\mathrm{s}}\right)\right) \text { for } \Theta \leq \frac{\pi}{2} \\
1.5+0.5 \tanh \left(8\left(\Theta-\frac{\pi}{2}-\Theta_{\mathrm{s}}\right)\right) \text { for } \Theta>\frac{\pi}{2}
\end{array}\right. \\
\text { with } \Theta_{\mathrm{s}}=\frac{20}{180} \pi,
\end{gathered}
$$

where $\Theta_{\mathrm{s}}$ is the angle that characterises the transition from low to high speed solar wind and thus specifies the inclination of the streamer belt with respect to the solar equator, as mentioned above. This angle is taken as $20^{\circ}$ in the following. Figure 2 shows the function $f_{\Theta}(\Theta) \cdot v_{0}$, which is symmetric to the heliographic equator. It shoud be pointed out that this structure occurs only at solar minimum conditions (see Sect. 3). To model the change from the solar wind structure at solar minimum to that at solar maximum, we suggest two models:

- Model 1: a first possibility is that the high solar wind speed beyond the streamer belt $\left(800 \mathrm{~km} \mathrm{~s}^{-1}\right)$ decreases during the increase of solar activity, until an average speed of $400 \mathrm{~km} \mathrm{~s}^{-1}$ is reached at all latitudes at solar maximum. 


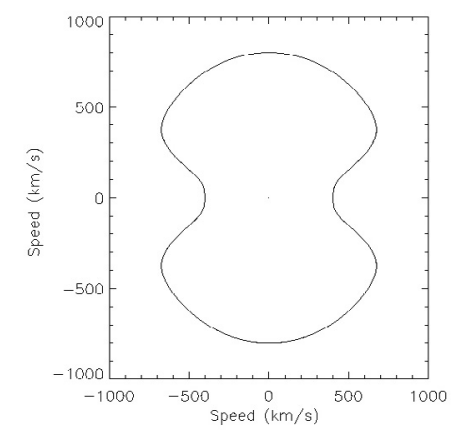

Fig. 2. The latitudinal structure of the solar wind velocity $f_{\Theta} v_{0}$, see Eq. (5).

- Model 2: a second possibility is, as mentioned already, that the inclination of the streamer belt of $20^{\circ}$ with respect to the solar equator increases with solar activity, until the angle has reached $90^{\circ}$ and the streamer belt extents over the whole Sun so that the solar wind speed is $400 \mathrm{~km} \mathrm{~s}^{-1}$ everywhere.

These two models are tested in this study. For the first model, the function $f_{\Theta}(\Theta)$ is modified in the following way:

$f_{\Theta} \cdot v_{0}=\left\{\begin{array}{l}V_{\min }+\frac{1}{2}\langle V\rangle \cdot\left(1-\tanh \left(x_{1}\right)\right) \cdot(1-\tanh (y(t))) \\ \text { for } \Theta \leq \frac{\pi}{2} \\ V_{\min }+\frac{1}{2}\langle V\rangle \cdot\left(1+\tanh \left(x_{2}\right)\right) \cdot(1-\tanh (y(t))) \\ \text { for } \Theta>\frac{\pi}{2}\end{array}\right\}(7)$

with:

$$
\begin{aligned}
x_{1} & =8\left(\Theta-\frac{\pi}{2}+\Theta_{\mathrm{s}}\right) \\
x_{2} & =8\left(\Theta-\frac{\pi}{2}-\Theta_{\mathrm{s}}\right) \quad \text { with } \Theta_{\mathrm{s}}=\frac{20}{180} \pi \\
y(t) & =g \cdot\left(t-t_{\mathrm{c}}\right) \\
\langle V\rangle & =\frac{1}{2}\left(V_{\text {max }}-V_{\min }\right),
\end{aligned}
$$

where $t$ is the time and $t_{\mathrm{c}}$ determines the point in time, when the decrease of the velocity is $50 \%$. The parameter $g$ enables the modeling of the duration of the transition period, for the change of the conditions of solar minimum into these of solar maximum. This parameter $g$ represents an interesting aspect of the modulation with reference to the solar activity cycle. $V_{\text {min }}$ and $V_{\max }$ have to be understood as the minimum and maximum solar wind velocity. Note, that $\langle V\rangle$ is not the average value, it rather represents a measure of the maximum velocity amplitude. For the second model, the inclination angle must be provided with a time-dependence:

$f_{\Theta} \cdot v_{0}=\left\{\begin{array}{l}V_{\max }-\langle V\rangle-\langle V\rangle \tanh \left(8\left(\Theta-\frac{\pi}{2}+\Theta_{\mathrm{s}}(t)\right)\right) \\ \text { for } \Theta \leq \frac{\pi}{2} \\ V_{\max }-\langle V\rangle+\langle V\rangle \tanh \left(8\left(\Theta-\frac{\pi}{2}-\Theta_{\mathrm{s}}(t)\right)\right) \\ \text { for } \Theta>\frac{\pi}{2}\end{array}\right\}$

$\Theta_{\mathrm{s}}(t)=\frac{20}{180} \pi+\left(1-\tanh \left(g \cdot\left(t_{\mathrm{c}}-t\right)\right)\right) \cdot \frac{23}{72} \pi$.

The inclination angle $\Theta_{\mathrm{s}}$ has to be increased up to $135^{\circ}$ $\left(90^{\circ}+45^{\circ}\right)$, because one has to make sure that the transition
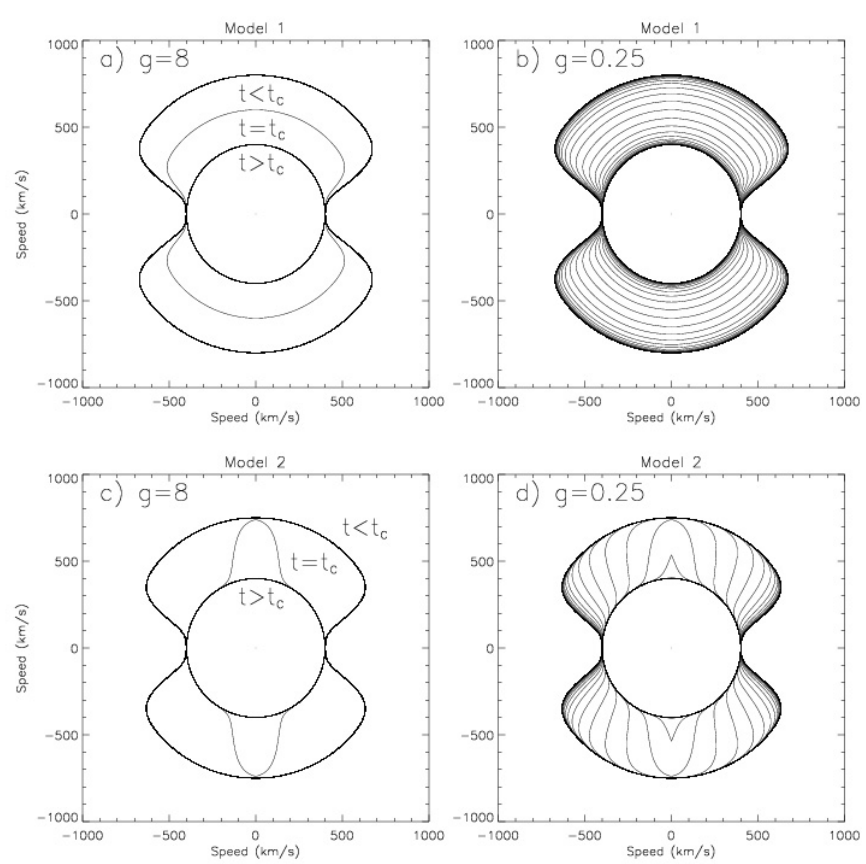

Fig. 3. The two time-dependent solar-wind models according to Eqs. (7) (Model 1) and (9) (Model 2) for different values of $g$. The parameter $g$ enables a modelling of the duration of the transition period, during which the conditions of solar minimum change into those of solar maximum. In a) and c) $g=8$, whereby only one time-step at $t=t_{\mathrm{c}}$ occurs. Thus, $g=8$ defines an upper limit for the parameter value $g$ considering the size of the time-step used in our computations. In b) and d) for $g=0.25$ the actual transition is resolved in several timesteps. Here we used $V_{\min }=400 \mathrm{~km} \mathrm{~s}^{-1}$ and $V_{\max }=800 \mathrm{~km} \mathrm{~s}^{-1}$.

region is taken into account, too. Figure 3 shows the two models for a parameter value $g=8$. With $g=8$ the transition is very quick, i.e. the velocity is decreased in just one timestep, where $t=t_{\mathrm{c}}$, panels a and c. If a smaller value for $g$ is chosen, the decrease takes more time, as shown in Fig. 3 for $g=0.25$, b and d. Both models arrive eventually at a uniform speed of $400 \mathrm{~km} \mathrm{~s}^{-1}$ at all latitudes. The solar wind velocity in the streamer belt region remains constant and has no timedependence. These two models will be tested in the numerical simulations with different values for $g$ to find out which one has more physical significance. Certainly, as a combination of the two suggested models is possible, too, also this is tested.

Before the electron flux simulations are considered, the two models can be compared with the SWOOPS-measurements at the time period from 1997 to 1999 , when solar activity was on the rise. Unfortunately, the Ulysses spacecraft was located at low latitudes during this period, namely in the region of the streamer belt, where the long-term averaged solar wind velocity has no time-dependence. Therefore, the models are not constraint by these data. If Ulysses would have been at high latitudes during this period, an evaluation of the two models and the transition period would have been possible under ideal conditions. Nonetheless, because of the sensitive interaction between convection and diffusion, the models can be tested via a comparison of solutions of the Parker-equation with UlyssesKET measurements. Particularly, the two models will also be 
used for the latitudinal diffusion, which has a dependence with on the activity cycle, too, as specified in the next chapter.

\section{The diffusion tensor}

The diffusion tensor used in Eq. (2) is given in spherical coordinates as:

$\kappa_{\text {polar }}=\left(\begin{array}{ccc}\kappa_{\|} \cos ^{2} \psi+\kappa_{\perp \mathrm{r}} \sin ^{2} \psi & 0 & \left(\kappa_{\perp \mathrm{r}}-\kappa_{\|}\right) \cos \psi \sin \psi \\ 0 & \kappa_{\perp \theta} & 0 \\ \left(\kappa_{\perp \mathrm{r}}-\kappa_{\|}\right) \cos \psi \sin \psi & 0 & \kappa_{\perp \mathrm{r}} \cos ^{2} \psi+\kappa_{\|} \sin ^{2} \psi\end{array}\right)$

and consists of a parallel diffusion coefficient $\kappa_{\|}$and two perpendicular coefficients, namely $\kappa_{\perp \mathrm{r}}$ in radial direction and $\kappa_{\perp \theta}$ in the polar direction. The spiral angle $\psi$ is defined as the angle between the radial direction and the average heliospheric magnetic field (HMF) at a certain position,

$\psi=\arctan \left(\frac{\omega}{u_{\mathrm{sw}}}\left(r-r_{0}\right) \sin \Theta\right)$ with $\omega=2.9 \times 10^{-6} \mathrm{~s}^{-1}$,

where $\omega$ is the angular velocity of the Sun, $u_{\mathrm{sw}}$ is the solar wind speed, $r_{0}=1 \mathrm{AU}$ and $\Theta$ is the polar angle.

\subsection{Diffusion coefficients}

For the parallel diffusion coefficient $\kappa_{\|}$, the following general form is assumed:

$\kappa_{\|}=\kappa_{0} \cdot \beta \cdot f_{1}(P, r)$,

where $\beta$ is the ratio of the speed of the electrons, $v$, to the speed of light, $c$, and $\kappa_{0}$ is a constant given as $4.5 \times 10^{18} \mathrm{~m}^{2} \mathrm{~s}^{-1}$.

$f_{1}$ is a function of the radial distance and the rigidity $P$ (or energy) for which we use the same functional dependence as given in Ferreira (2001a,b).

For the perpendicular diffusion coefficients it has become standard practice to scale these as the parallel coefficient (see e.g. Ferreira 2001a,b). Here the perpendicular coefficients $\kappa_{\perp \mathrm{r}}$ and $\kappa_{\perp \theta}$ are assumed to have the following form:

$\kappa_{\perp \mathrm{r}}=\delta(P) \cdot \kappa_{\|}$,

$\kappa_{\perp \Theta}=\epsilon \cdot \kappa_{\|} \cdot F(\Theta)$,

where $\kappa_{\perp \mathrm{r}}$ has an additional dependence on rigidity $P$ :

$\delta(P)=0.02\left(\frac{P}{P_{0}}\right)^{0.3}, \quad \epsilon=0.015, \quad P_{0}=1 \mathrm{GV}$

and

$F(\Theta)=\left\{\begin{array}{l}\frac{d+1}{2}+\frac{d-1}{2} \cdot \tanh \left(8\left(+\frac{\pi}{2}-\Theta-\Theta_{s}\right)\right) \text { for } \Theta \leq \frac{\pi}{2} \\ \frac{d+1}{2}+\frac{d-1}{2} \cdot \tanh \left(8\left(-\frac{\pi}{2}+\Theta-\Theta_{s}\right)\right) \text { for } \Theta>\frac{\pi}{2},\end{array}\right.$

where $\Theta_{\mathrm{s}}$ is once again the angle, which characterises the inclination of the streamer belt. Equation (17) is shown in Fig. 4 as a function of polar angle with three different values for $d$, for which the subsequent effects on the modulation will be studied in this work. Figure 4 illustrates how $F(\Theta)$ increases from unity in the ecliptic plane $\left(90^{\circ}\right)$, which gives no enhancement, towards the poles depending on the value of $d$.

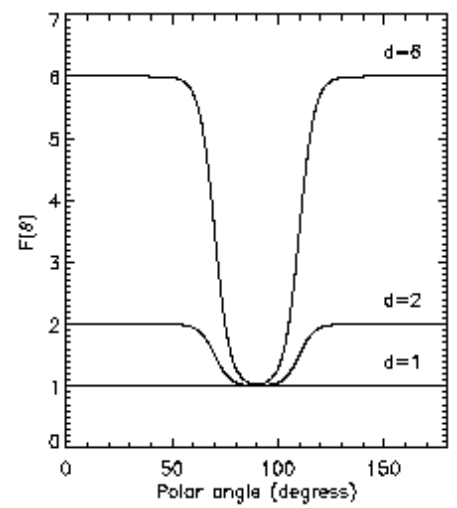

Fig. 4. $F(\Theta)$ for three different values of the parameter $d$.

Ferreira et al. (2001a) demonstrated that in the phase of solar minimum an enhanced latitudinal transport corresponding to $d>1$ is necessary. This is accomplished by increasing $\kappa_{\perp \theta}$ toward the poles by a factor of $d=6$ with respect to the value in the equatorial plane, see Fig. 4.

Burger et al. (2000) give a physical justification of this increase in $\kappa_{\perp \theta}$ on the basis of Ulysses measurements that show the variance to increases more in the transverse and normal directions of the HMF than in the radial direction, resulting in stronger diffusion in these directions. Furthermore, for a Fisktype HMF (Fisk 1996), which probably has a more realistic geometry, latitudinal transport is supposedly more effective than in a Parker field (which is used in the numerical model), and to account for this effect $\kappa_{\perp \theta}$ is enhanced toward the polar regions.

In solar maximum conditions, however, a reduction in the enhancement of latitudinal transport towards the poles from its solar minimum value is necessary (see Henize et al. 2003). This can be correlated to the disappearance of the fast solar wind and/or the vanishing of a meridional component of the HMF during solar maximum. Analogously to the variation of the latitudinal structure of the solar wind it is assumed that the factor $d=1$ applies to all latitudes at solar maximum.

Because of the analogous form of the latitudinal structure of solar wind (Eq. (5)) and the function $F(\Theta)$ (Eq. (17)) it is evident that the two proposed models for the latitudinal variation of the solar wind can be used for the variation of the perpendicular latitudinal diffusion coefficient as well. Therefore, in the first model the parameter $d$ and in the second the inclination angle $\Theta_{\mathrm{s}}$ are provided with a time-dependence as follows:

$d(t)=6-2.5 \cdot(1-\tanh (y(t)))$,

$\Theta_{\mathrm{s}}=\frac{20}{180} \pi$ for Model 1

$\Theta_{\mathrm{s}}(t)=\frac{20}{180} \pi+(1-\tanh (y(t))) \cdot \frac{23}{72} \pi$,

$$
d=6 \text { for Model } 2
$$

with

$y(t)=g \cdot\left(t_{\mathrm{c}}-t\right)$.

As before, the parameter $g$ allows for a modeling of the transition period, over which the conditions of solar minimum $(d=6)$ change into the conditions of solar maximum $(d=1)$. 


\section{KET-observations and comparison with simulations}

The time-dependent latitudinal diffusion coefficient together with the time-dependent latitudinal solar wind structure were incorporated into the Parker-equation and the two models were tested in the numerical simulations with different values for $g$. The model computations were then compared to the electron intensities observed by the KET/Ulysses instrument. To solve the Parker-equation we use the VLUGR3 code developed by Blom \& Verwer (1994a,b).

\subsection{Modeling the heliosphere}

The outer boundary of the simulated heliosphere was set to be at $120 \mathrm{AU}$, where the electron spectrum determined by Langner et al. (2001) was used as the local interstellar spectrum for galactic electrons. The source function $j_{\mathrm{s}}$ for electrons produced at Jupiter is given by (Ferreira 2001a)

$j_{\mathrm{s}}=1.5\left(\frac{c_{\mathrm{k}} j_{1.5} d_{\mathrm{k}} j_{6.0}}{c_{\mathrm{k}} j_{1.5}+d_{\mathrm{k}} j_{6.0}}\right)$,

with

$j_{6.0}=10^{9} E^{-6.0}, \quad c_{\mathrm{k}}=0.6, \quad d_{\mathrm{k}}=5.0$.

This function, with differential intensity $j_{\mathrm{s}}$, given in units of $\mathrm{m}^{-2} \mathrm{sr}^{-1} \mathrm{~s}^{-1} \mathrm{MeV}^{-1}$ and kinetic energy in units of $\mathrm{GeV}$, is a combination of $j \sim E^{-1.5}$ and $j \sim E^{-6.0}$ spectra and is constructed to be compatible with ISEE 3 spectra and Pioneer 10 data (Ferreira 2001a). The source is implemented in the numerical model as a point source by specifying the source function at a single grid point with an extent of $\sim 0.15 \mathrm{AU}$ in radial direction. Especially the large scale time evolution in the electron intensity is of note, why effects of the Sun rotation, e.g. CIRs (Corotating Interaction Regions), are not taken into account. For modelling the influence of a CIR on the propagation of Jovian electrons see Kissmann (2002, 2004).

\subsection{Time-independent simulation}

At first, a time-independent test of the numerical model is presented. This means that a simulation was conducted without a time-dependence of the solar wind speed $u_{\mathrm{sw}}$ and the latitudinal diffusion coefficient $\kappa_{\perp \Theta}$. The latitudinal structure of the solar wind and the diffusion is kept at the conditions valid for solar minimum. The computed intensities along the Ulysses trajectory are shown in Fig. 5. In addition, the observed 3-10 MeV Ulysses/KET data are displayed in gray. The radial and latitudinal coordinate of the Ulysses trajectory are shown at the top panel.

In February 1992, the Jupiter fly-by of the Ulysses spacecraft is evident by the high intensities then. Since this fly-by, the spacecraft is travelling in an elliptical, Sun-focused orbit inclined by 80 degrees w.r.t. the solar equator. After the first passage through the ecliptic plane in 1995 the evolution of the computed intensities differs markedly from the KET-data.

This is due to the fact that there is no time-dependence in this simulation. Apparently the change of solar activity

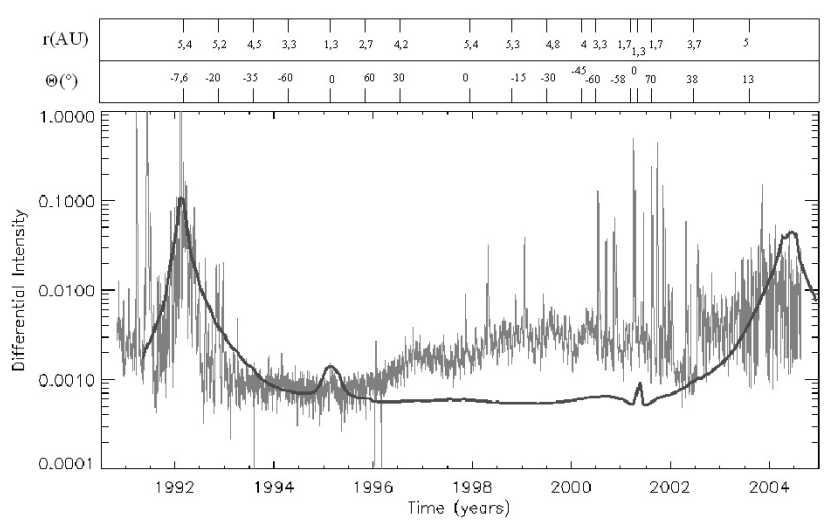

Fig. 5. The computed electron intensities along the Ulysses trajectory at an energy of $7 \mathrm{MeV}$ (solid line). In addition, the Ulysses/KET data are plotted. This simulation was performed without a time-dependence of the solar wind speed $u_{\mathrm{sw}}$ and the latitudinal (perpendicular) diffusion coefficient $\kappa_{\perp \Theta}$. The latitudinal structure of the solar wind and the diffusion is kept at conditions valid for solar minimum. The radial and latitudinal coordinate of the Ulysses trajectory are shown at the top panel. Overview of the parameters used: $V_{\min }=400 \mathrm{~km} \mathrm{~s}^{-1}$, $V_{\max }=750 \mathrm{~km} \mathrm{~s}^{-1}, d=6$.

conditions affects the transport processes from the year $\sim 1996$ onwards. In the beginning of the year 2001 another ecliptic passage took place, which is distinct from the passage in 1998, because at this time Jupiter was, seen from Ulysses, behind the Sun, so that the Jovian electron source was largely shielded. In the year 2004, 12 years after the first encounter with Jupiter, the second fly-by of the Ulysses spacecraft occurred. It was, however, at a seven times larger distance than the first one, which is reflected by the lower intensity at that time. This timeindependent simulation run represents a reference for the timedependent runs, which will be discussed in the following.

\subsection{Time-dependent simulations and variation of the Jovian source}

For the following results the suggested time-dependent parameters were implemented in the simulation. First of all, model 1 was used for the solar wind (Eqs. (7), (8)) and the latitudinal diffusion (Eqs. (17), (18)) with a parameter value of 8 for $g$.

Thus, the transition period, with the conditions of solar minimum changing into those of solar maximum, corresponds to just one time-step. The parameter $t_{\mathrm{c}}$, which determines the instant, when the decrease of the solar wind and the latitudinal diffusion is 50\%, is chosen as 1995.8. The solid line in Fig. 6 shows the results for this simulation. Up to 1996 the calculated electron intensities comply with the intensities in Fig. 5, because the time-dependence is moderate not until $t_{\mathrm{c}}=1995.8$. During solar maximum conditions from onwards $\sim 1996$ the simulated intensities are higher than in the time-independent simulation, Fig. 5, caused by the reduced solar wind velocity and latitudinal diffusion. Thereby, the convection speed has decreased to almost half of the solar minimum value, so that the energetic electrons are not any longer strongly transported outwards. In addition, the latitudinal diffusion at high latitudes is 


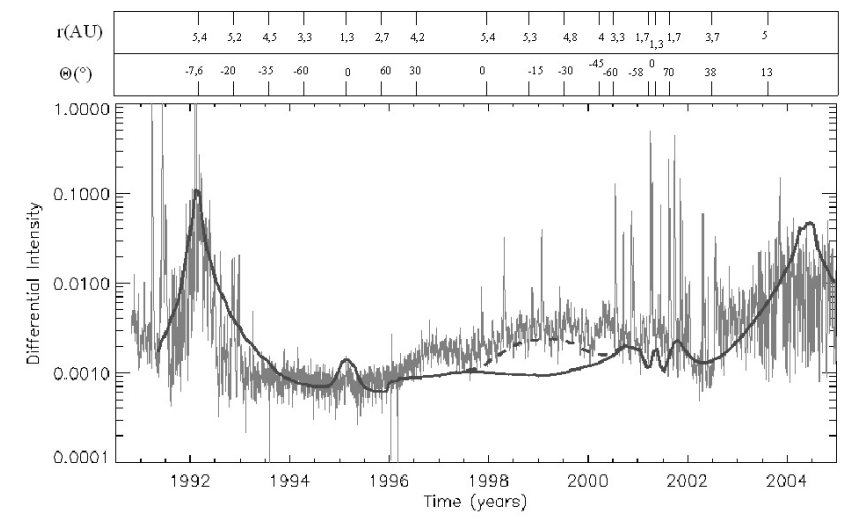

Fig. 6. Two simulations using a set of time-dependent parameters. Model 1 is used for the solar wind (Eqs. (7), (8)) and the latitudinal (perpendicular) diffusion (Eqs. (17), (18)) with the parameter $g=8$. From simulation with the solid line it is evident that the calculated intensities in the period of 1998 to 2001 are much lower than the $7 \mathrm{MeV}$ Ulysses/KET data. In the simulation with the dashed line, therefore, we implemented an increase of the Jovian source strength in form of a Gaussian with a maximum in mid 1999 and an increase of the source strength by a factor of 8 . The solid and dashed line have only a different evolution from $\sim 1998$ to $\sim 2000$. Overview of the parameters used: $V_{\min }=400 \mathrm{~km} \mathrm{~s}^{-1}, V_{\max }=750 \mathrm{~km} \mathrm{~s}^{-1}, t_{\mathrm{c}}=1995.8, d=6$.

reduced to the value of the streamer belt, so that the Jovian electrons can propagate more homogeneously to all heliographic latitudes. The change in the latitudinal structure of solar wind and diffusion happens very quickly, and consequently, the computed intensities rise rapidly in $\sim 1996$.

Note that the conditions of solar maximum, which from since 1996 onwards, were valid up to the end of the simulation. A distinct decrease in solar activity is not expected until mid 2005. A comparison with KET data gives the following insight: The computed electron intensities increase indeed from 1996, but the increase in the data is stronger and slower. Especially in the period of 1998 to 2001 the simulations produce much lower intensities than those measured by Ulysses. One possibility of this electron lack is a too strong diffusion in latitudinal and/or radial direction. This will be discussed later.

Since the Ulysses spacecraft is in the inner heliosphere, the dominant electron source is the Jovian source, so that a second possibility is a variability of the Jovian source strength in this period. Morioka et al. (1999) have deduced a variation of the Jovian source strength under reference to Ulysses measurements. Thereupon Henize et al. (2003) have studied the effect of a variation of the Jovian source strength in steadystate simulations. They utilise an increase of the source strength in form of a Gaussian curve with a maximum in 1999 and maximum increase of the source strength by a factor of 2.5. In combination with a lower latitudinal diffusion in the period of solar maximum the authors achieved results compatible with KET/Ulysses data. This suggested variation of the Jovian source strength by Henize et al. (2003) was added to the time-dependent simulation here. The results are shown as the dashed line in Fig. 6. In this simulation the source strength is increased by a factor of 8 , because it turned out that an increase of a factor 2.5 is insufficient here. Apparently, by a

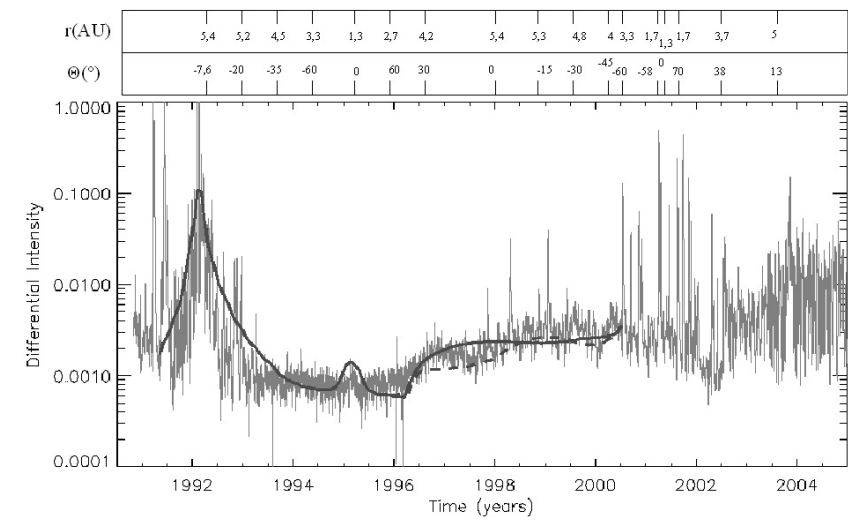

Fig. 7. The dashed line shows a simulation, where the latitudinal (perpendicular) diffusion coefficient is reduced to $50 \%$ from $t_{\mathrm{c}}$ onwards in comparison to the value before $t_{\mathrm{c}}$. For the solid line the radial (perpendicular) diffusion coefficient is increased to $35 \%$ from $t_{\mathrm{c}}$ onwards. In both simulations the increase of the Jovian source strength by a factor of 8 is taken into account. The simulations stop at 2000.5, because only the time frame of the Jovian source variation is of interest here. In addition, the $7 \mathrm{MeV}$ Ulysses/KET data are plotted. Overview of the parameters used: $V_{\min }=400 \mathrm{~km} \mathrm{~s}^{-1}, V_{\max }=750 \mathrm{~km} \mathrm{~s}^{-1}, t_{\mathrm{c}}=1996.2$, $d=6$.

significant variation of the Jovian source strength one is able to reproduce the Ulysses/KET data also in the period of $\sim 1998$ to $\sim 2000$. The ultimate cause of the variable source strength is largely unknown. Morioka et al. argue that it comes from the variable solar wind pressure at the Jovian magnetosphere. (For more details see Morioka et al. 1999). Kanekal et al. (2003) observed also a variable Jovian source strength in $\sim 1997$, using instruments on board the SAMPEX and IMP8 spacecrafts at 1 AU. As mentioned above, a modification of the diffusion coefficients can help to increase the electron intensities in the inner heliosphere. In combination with the variable Jovian source strength it may produce even better results and permit a lower increase of the Jovian source strength than factor of 8 . This is discussed below.

\subsection{Modification of the diffusion coefficients}

At first it was tested, whether a lower latitudinal diffusion created the possibility of a variation of the Jovian source strength around 1999 of less than a factor of 8 . A less efficient diffusion of the electrons in latitudinal direction will bring about an increase in the intensity at low latitudes, because the dominant electron source, Jupiter, lies in the ecliptic.

The dashed line in Fig. 7 shows results of a simulation, where the latitudinal diffusion coefficients is reduced to $50 \%$ from $t_{\mathrm{c}}=1996.2$ onwards. Due to the use of the lower latitudinal diffusion coefficient a distinct increase in intensity form $\sim 1996$ is visible in contrast to Fig. 6. It is remarkable that electrons are apparently gathering at high latitudes as well: these electrons are galactic cosmic rays. Because of the reduced latitudinal diffusion the uniform repartition of the electrons is now very inefficient in this direction. During the period of the 8-times stronger Jovian source $(\sim 1999)$ no essential increase in the intensity is identifiable. This is due to the fact, 
that Jupiter was at this time, seen from Ulysses, behind the Sun, so that the Jovian electron source was largely shielded. The reduced latitudinal diffusion (even at about 50\%) has, consequently, no effect on the electron distribution at and near the position of Ulysses during this period. The significant variation of the Jovian source strength is still necessary to reproduce the Ulysses/KET data in the period of $\sim 1998$ to $\sim 2000$.

A second possibility is an increase of the radial diffusion. Through this, the electron intensity can be raised in the entire inner heliosphere. To modify the radial diffusion coefficient, it must be pointed out that it has a special effect in the outer heliosphere. Ferreira (2001b) was able to fit the radial diffusion coefficient using Pioneer 10 data in the outer heliosphere. The Poineer 10 spacecraft has measured the electron intensities up to a distance of 70 AU. Ferreira's calculations show that an increase of the radial diffusion coefficient by more than $35 \%$ is not acceptable, because the outer heliosphere would otherwise "overload" with electrons. By the solid line of Fig. 7 we picture a simulation, in which the radial diffusion is increased by $35 \%$ from $t_{\mathrm{c}}=1996.2$ onwards. As expected, a dramatic increase in intensity from $\sim 1996$ is visible, which is mainly due to Jovian electrons. The electron intensity from $\sim 1997$ to $\sim 2001$ is nearly constant, because of the increased radial diffusion the Jovian source fills the heliosphere nearly isotropically. Because of this and the fact that the Jovian source was, as mentioned, largely shielded seen from Ulysses at the period of the increased Jupiter source, the dramatic variation of the source strength $\sim 1999$ is admittedly not identifiable. It should be noted that the Jovian source is not implemeted as a source term in the Parker-Equation, but exists as a fixed value for the electron-flux in the numerical grid-system.

The following results can be stated: the increase of the radial diffusion brings higher intensities in the heliosphere, but permits no lower increase of the Jovian source strength than a factor of 8 . Neither via the modification of the latitudinal diffusion nor of the radial diffusion allow to avoid the dramatic increase of the Jovian source strength. Therefore, the increase of the source strength by a factor of 8 in form of a Gaussian is used in the subsequent simulations as well. In the following chaper the parameter study with the two suggested models will commence. So far, only the first model with a parameter value of 8 for $g$ was used.

\subsection{Time-dependent solar wind and diffusion models}

The upper panel of Fig. 8 displays a simulation using the first model, with a time dependent solar wind velocity and ratio of the latitudinal diffusion, respectively. For this, the solar wind velocity is varied from $V_{\max }=750 \mathrm{~km} \mathrm{~s}^{-1}$ to $V_{\min }=400 \mathrm{~km} \mathrm{~s}^{-1}$ at high latitudes. The parameter $d$ of the latitudinal diffusion is varied from $d=6$ at high latitudes to $d=1$ at all latitudes. The parameter $g$, that determines the duration of the transition period, is chose equal to 8 , so that the transition is very sharp. In addition, the radial diffusion is increased about $15 \%$ form $t_{\mathrm{c}}=1995.8$ onwards. As mentioned, the variation of the Jovian source strength by a factor of 8 around $\sim 1999$ is implemented, too.

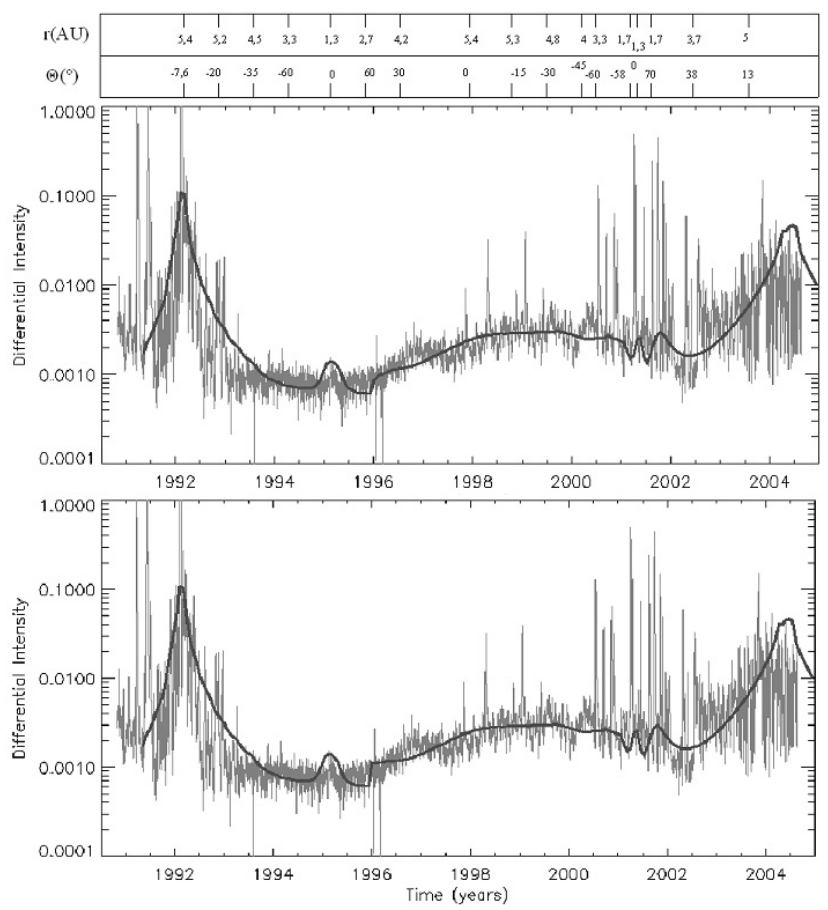

Fig. 8. The upper panel displays a simulation using the model 1, the lower panel results from model 2 . The parameter $g$, that determines the transition period, is chosen for both equal to 8 , so that the transition is very sharp. The two simulations show a nearly identical evolution of the electron intensity. In addition, the $7 \mathrm{MeV}$ Ulysses/KET data are plotted. Overview of the parameters used: $V_{\min }=400 \mathrm{~km} \mathrm{~s}^{-1}, V_{\max }=$ $750 \mathrm{~km} \mathrm{~s}^{-1}, t_{\mathrm{c}}=1995.8$.

The lower panel of Fig. 8 displays a simulation using the second model but with the same parameters. Here the inclination of the streamer-belt of $20^{\circ}$ compared to the solar equator is increased, until the minimum speed $V_{\text {min }}=400 \mathrm{~km} \mathrm{~s}^{-1}$ and $d=1$ exist at all latitudes. The two simulations in Fig. 8 show, however, the nearly identical evolution of the electron intensities. This was expected, because in both models the changes in the simulation occur in just one time step. The changes in the solar wind velocity and latitudinal diffusion go on so quickly, that no essential differences are noticeable.

If the parameter value is chosen smaller than 8 , the transition period, during which the solar minimum conditions change into maximum, is increased. A parameter value of $g=4,2,1, \frac{1}{2}, \frac{1}{4}, \frac{1}{8}$ or $\frac{1}{16}$ is essentially identical $g=8$ case (Fig. 8). Not until $g=\frac{3}{32}$ a different evolution occurs. This value correspond to 19 time steps, or 1.4 years. Figure 9 shows the results for the first model (solid line) and the second model (dashed line). For the first model (solid line) the increase in intensity is no longer as rapid as in Fig. 8. The intensity increases here one a period of $\sim 1$ year (1996-1997). This is expected, because the convection, just as the latitudinal diffusion, decreases slower at high latitudes than in Fig. 8 with $g=8$. In the time interval of this change the Ulysses spacecraft covers a latitudinal range of nearly $60^{\circ}$. In the second model (dashed line) occurs a local maximum in $\sim 1996$, whereas the increase ends at higher intensities than in Fig. 8 (with $g=8$ ). This can be understood by Fig. 11, where the position of the Ulysses 


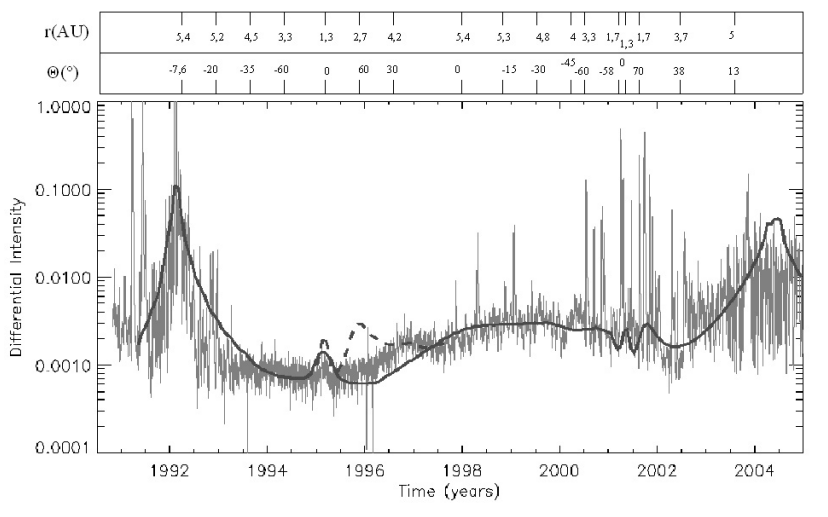

Fig. 9. Two simulations showing the results for a parameter value of $g=\frac{3}{32}$ for the model 1 (solid line) and the second model (dashed line). This value specifies 19 time steps, corresponding to 1.4 years. The intensity evolution of the two simulations shows clear differences for the two models. In addition, the $7 \mathrm{MeV}$ Ulysses/KET data are plotted. Overview of the parameters used: $V_{\min }=400 \mathrm{~km} \mathrm{~s}^{-1}$, $V_{\max }=750 \mathrm{~km} \mathrm{~s}^{-1}, t_{\mathrm{c}}=1995.8$.

spacecraft together with the inclination angle $\Theta_{\mathrm{s}}(t)$ for three different points in time is shown. In addition, the values for the solar wind speed and the latitudinal diffusion parameter $d$ are given. The left panel shows the situation during the ecliptic passage 1995. About the same time the change of $\Theta_{\mathrm{s}}(t)$ gets in. This angle of $20^{\circ}$ increases now and moves to higher heliographic latitudes. The "numerical" Ulysses spacecraft trails this increasing angle, until it overtakes it in $~ 1996$. In this period (1995 till 1996) occurs the increase and maximum of the simulated electron intensity (dashed line in Fig. 9). Indeed the simulation provides clearly more electrons than measured by Ulysses. In the beginning of 1996 the position of the spacecraft and $\Theta_{\mathrm{s}}(t)$ coincide at about $\sim 55^{\circ}$ (see middle panel in Fig. 11). From then on the "numerical" spacecraft flew faster to high latitudes as the inclination angle $\Theta_{\mathrm{s}}(t)$ (whose "velocity" can be controlled by the parameter $g$ ). Thus, after 1996 the spacecraft was located in the region, where the conditions of the solar minimum were still prevailing. Therefore, the electron intensity decreased after 1996. Not until the spacecraft and $\Theta_{\mathrm{s}}(t)$ met each other again at $\sim 1996.3$ (see right panel in Fig. 11), the electron intensity decrease stopped. After 1996.5 the inclination angle has reached $90^{\circ}$ and the conditions of the solar maximum existed now at all latitudes und then the calculated intensities in Fig. 9, dashed and solid line, are identical. Furthermore, it must be pointed out that the evolution of the electron intensity during the ecliptic passage in 1995 is different in Fig. 8 (lower panel) and Fig. 9 (dashed line), although in both model 2 is in use (with parameter values $g=8$ and $g=\frac{3}{32}$ ).

This is in contrast to Fig. 8 (upper panel) and Fig. 9 (solid line), where the evolution of intensity is identical and the model 1 is in use. Apparently, different values of $g(g=8$ and $g=\frac{3}{32}$ ) affect the electron distribution in the ecliptic plane only if the second model is used. This differences are caused by the successive enlargement of the streamer belt in the second model, whereas in the first model the conditions at high latitudes are successively brought into line with the streamer belt conditions. In fact, the inclination angle with $g=\frac{3}{32}$ increases very slowly, but the transition area is unchanged, sharp and strongly confined. Through this, the changes in convection and in diffusion appear quickly, such that the local maximum 1996 occurs. Consequently, the model 1, with the solar wind speed and latitudinal diffusion at high latitudes brought into line with the streamer belt conditions, proves here as a suitable model. In $\sim 1995.5$, where the time-dependences of the two models is in operation, Ulysses passes the inclination angle of $20^{\circ}$ heliographic latitude, which is time-independent in the first model. In the solid line of Fig. 9 (first model) there is, indeed, no transition identifiable in contrast to the dashed line (second model) where during transit of the inclination angle (at about at $~ 55$ ) an increase of the intensity appears. This shows once more, that the first model with the time-independent inclination angle $\Theta_{\mathrm{s}}$ is more appropriate than the second model with $\Theta_{\mathrm{s}}(t)$.

There is also the possibility of a combination of the two models. This means that the velocity of the solar wind and the parameter $d$ of the latitudinal diffusion are chosen to be time-dependent with an additional variation in the inclination angle $\Theta_{\mathrm{s}}(t)$. But from the above results it is clear, that a combination of the two models brings no essential advantages. A simulation with a combination shows nearly a combined evolution of the two distributions in Fig. 9. Because of this, the first model will be considered in the following only. Another interesting aspect is a different transit period for the solar wind and the latitudinal diffusion. Up to now for the solar wind and the diffusion coefficient we used the same value $g$. If different values of $g$ are be used, the complex interaction of convection and diffusion come to the fore, while the diffusion is the most effective transport process of energetic electrons. A higher value for $g$ in the diffusion than in the solar wind produces strong gradients in the electron intensity. In contrast, a higher value for $g$ in the solar wind than in the latitudinal diffusion produces results, which are comparable with the previous simulations. Figure 10 displays a simulation, where $g=1 / 2$ for the solar wind and $g=1 / 4$ for the latitudinal diffusion. That means, the latitudinal solar wind structure reached twice as rapidly the solar maximum conditions as the latitudinal diffusion. A parameter value of $g=1 / 2$ complies with a period of about two months, $g=1 / 4$ with $\sim$ four months, accordingly. This transition period is once again very sharp (as opposed to Fig. 9 with $g=3 / 32$ ), but the results in Fig. 10 show no strong rise in intensity, because the diffusion there takes more time to reduce, so that the electrons are slow-going to higher latitudes, where the convection has already reached the minimal value. In addition, we have also tested different values for the maximal and minimal solar wind speed. The best results we obtain by using $V_{\max }=800 \mathrm{~km} \mathrm{~s}^{-1}$ and $V_{\min }=350 \mathrm{~km} \mathrm{~s}^{-1}$, while in the above simualtions $V_{\max }=750 \mathrm{~km} \mathrm{~s}^{-1}$ and $V_{\min }=400 \mathrm{~km} \mathrm{~s}^{-1}$ was used. Therewith only the quantity $\langle V\rangle$ (see (8)) is increased, but the average value $\langle V\rangle+V_{\text {min }}$ remains constant.

The results in Fig. 10 represent with these parameter values so far the best approximation on the Ulysses/KET data. Especially notable is the temporary drop of intensity $\sim 1997$, which is also seen in the KET data. Figure 12 displays the situation in the meridional plane at 1997.0, where contours are shown for the electron intensities. Near to the ecliptic plane 


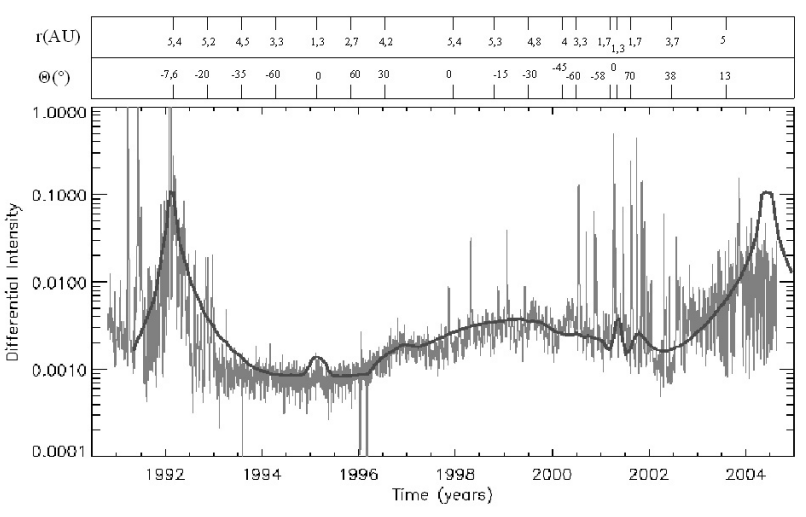

Fig. 10. In this simulation different transit periods were used for the solar wind and the latitudinal (perpendicular) diffusion. Here for the solar wind $g=1 / 2$ and for the latitudinal diffusion $g=1 / 4$. In addition, the maximal solar wind speed is chosen as $V_{\max }=800 \mathrm{~km} \mathrm{~s}^{-1}$ and the minimal as $V_{\min }=350 \mathrm{~km} \mathrm{~s}^{-1}$. The intensity results represent so far the best approximation on the $7 \mathrm{MeV}$ Ulysses/KET data. Overview of the parameters used: $V_{\min }=350 \mathrm{~km} \mathrm{~s}^{-1}, V_{\max }=800 \mathrm{~km} \mathrm{~s}^{-1}$, $t_{\mathrm{c}}=1995.8$.
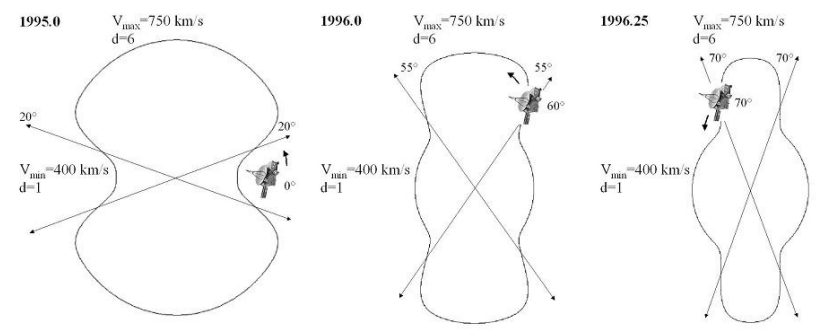

Fig. 11. Latitudinal position of the Ulysses spacecraft together with the inclination angle $\Theta_{\mathrm{s}}(t)$ of model 2 for three different points in time. In addition, the values for the solar wind speed and the latitudinal diffusion parameter $d$ are denoted. The left panel shows the situation during the ecliptic passage 1995. About the same time the timedependent $\Theta_{\mathrm{S}}(t)$ starts to shift the transition region. The evolution of the electron intensity form Fig. 9 (dashed line) can be understood with this illustration (see text).

the effects of the Parker spiral and the extensions of the Jovian source are visible. The spacecraft was located at $20^{\circ}$ heliographic latitudes and the conditions of solar maximum were already existent at all latitudes. But evidently the spacecraft was located at a local minimum of electron intensity. At higher latitudes the intensity increases again, but in a relatively small band. In fact, the same solar wind speed $\left(350 \mathrm{~km} \mathrm{~s}^{-1}\right)$ and the same value of latitudinal diffusion (with $d=1$ ) is existent at all latitudes, but the electrons need, especially in latitude, more time to reach uniform distribution. This behaviour certainly occurs in all simulations above, but is essentially boosted by the different transition periods together with the increased solar wind velocity gradient. This local minimum is seen in the KET data as well. Furthermore, the intensity at the second Jupiter flyby in 2004 is increased in comparison to the other simulations above. This is expected, because the convection at the location of the Jovian source is decreased due to the lower minimum solar wind speed $\left(V_{\min }=350 \mathrm{~km} \mathrm{~s}^{-1}\right)$. In the period of solar maximum in about 2000 an exact modelling of the

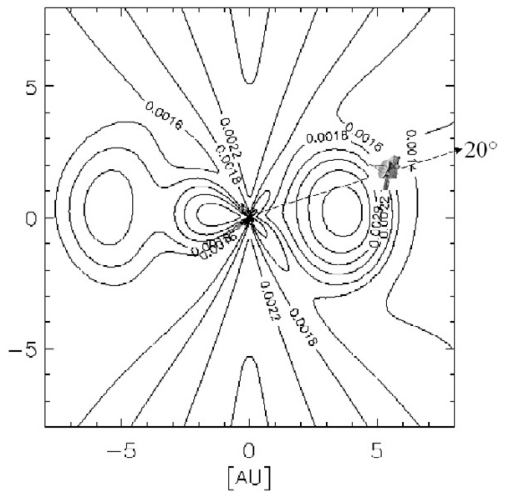

Fig. 12. Electron intensity distribution in the meridional-plane at 1997.0 for Fig. 10. The spacecraft was located at a heliographic latitude of $20^{\circ}$. Near to the ecliptic plane effects of the Parker spiral and the extensionss of the Jovian source are visible.

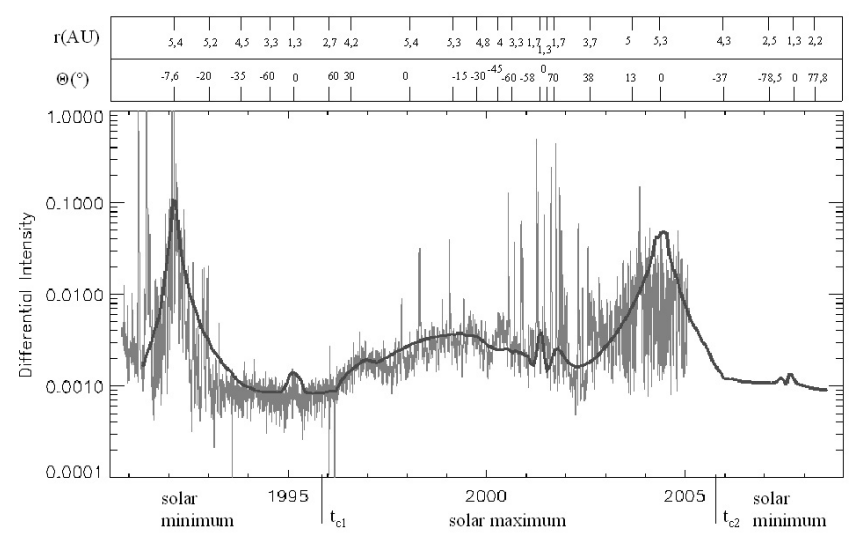

Fig. 13. This simulation represents an outlook until 2008. The phases of the solar activity are marked. The parameter $t_{\mathrm{c} 2}$ for the declining phase of solar activity is assumed to be 2005.8 . In addition, the $7 \mathrm{MeV}$ Ulysses/KET data until 2005 are plotted. Overview of the parameters used: $V_{\min }=350 \mathrm{~km} \mathrm{~s}^{-1}, V_{\max }=800 \mathrm{~km} \mathrm{~s}^{-1}, t_{\mathrm{c} 1}=1995.8, t_{\mathrm{c} 2}=$ 2005.8 .

measured intensity is very difficult or impossible, because of the high solar activity with its associated variability in the electron intensity. Certainly, in connection with the solar activity there are more complicated time-dependences imaginable but the principle intensity characteristics, especially in the transition period from solar minimum to maximum conditions ( $\sim 1995$ to $\sim 2000)$, can be reproduced very well with a relatively simple time-dependence as demonstrated above.

\section{Outlook}

In February 2004 the Ulysses-Mission was extended until March 2008. Then the operating time will have reached about 17 years and in December 2006 the spacecraft will pass the Sun for the third time. Thus, Ulysses will be measuring the next solar minimum, which should begin at the end of 2005 .

Figure 13 shows a simulation, which runs to mid 2008. There we used the same parameters as in Fig. 10. In addition, the transition period from solar maximum to the minimum is set to 2006 . The phases of the solar activity are marked. The parameter $t_{\mathrm{c} 2}$ for the declining phase of solar activity is 
assumed to be 2005.8. It is visible, that the peak of the second Jupiter fly-by in 2004 starts much earlier in comparison to the first one in 1992. This broadening in the electron intensity is due to in the fact, that this passage occurs into the period of solar maximum. The Jovian electrons can easier reach higher latitudes because of the lower convection and diffusion there. After $t_{\mathrm{c} 2}=2005.8$, the intensity reaches the same level as before in $t_{\mathrm{c} 1}=1995.8$, because the solar minimum conditions exist once again. In the middle of 2007, another ecliptic passage occurs. It remains to be seen, whether or not the Ulysses/KET data will confirm these predictions for the electron intensity trend.

\section{Summary}

We used a time-dependent 3D-model to simulate the $7 \mathrm{MeV}$ electron fluxes in the heliosphere under special consideration of the influence of the solar activity cycle. On the basis of the Ulysses/SWOOPS measurements of the latitudinal solar wind structure we have suggested and formulated two models, which characterise the varying velocity field of the solar wind with the solar cycle. In addition it is shown, that these models can be implemented in a time-dependent latitudinal diffusion coefficient as well.

From a comparison with Ulysses/KET data it turned out, that the first model (see Eq. (7)), which brings the solar wind speed and the value of latitudinal diffusion at high latitudes into line with the streamer belt conditions, proves as suitable. The second model (see Eq. (9)), which increased the $20^{\circ}$ inclination of the streamer-belt with the solar equator, leads to higher electron intensities, which are not in line with the KET data. In addition it was shown, that a significant variation of the Jovian source strength is necessary in the period of $\sim 1998$ to $\sim 2000$. This variation is implementated in form of a Gaussian curve with a maximum in 1999 and maximum increase of the source strength by a factor of 8 . A modification of the latitudinal or radial diffusion coefficient $\kappa_{\perp \mathrm{r}}$ and $\kappa_{\perp \theta}$ was tested, but permits no possibility of a lower increase of the source strength.

The best fit to the Ulysses/KET data can be reached with different transit periods for the solar wind and the latitudinal diffusion. If the latitudinal solar wind structure reached twice as rapidly the solar maximum conditions as the latitudinal diffusion $\sim 1996$, the evolution of the computed electron intensities can in good approximation reproduce the KET-data. In doing so, the transit period for the solar wind and the latitudinal diffusion were found to be about two and about four months, respectively.

The principle electron intensity characteristics, especially in the transition period from solar minimum to maximum conditions ( $\sim 1995$ to $\sim 2000)$, can be reproduced very well with these relatively simple models for the time-dependences. In the period of solar maximum 2000 exact modelling is very difficult or impossible due to the high solar activity and the accompanying variabilities in the solar wind speed and the electron intensity. The Ulysses-Mission was extended until March 2008, so that the modelling of the KET data will remain an interesting and valuable research field for years.

Acknowledgements. We thank PD Dr. Bernd Heber for providing the Ulysses/KET data and for critical discussion. The work benefited from UCRJET collaboration supported by the Deutsche Forschungsgemeinschaft (DFG) and the South African National Research Foundation (NRF).

\section{References}

Blom, J. G., \& Verwer, J. G. 1994a, VLUGR3: A vectorizable adaptive grid solver for PDEs in 3D I, Algorithmic aspects and applications, Report NM-R9404, CWI, Amsterdam

Blom, J. G., \& Verwer, J. G. 1994b, VLUGR3: A vectorizable adaptive grid solver for PDEs in 3D II, Code Description, Report NMR9405, CWI, Amsterdam

Burger, R. A., Potgieter, M. S., \& Heber, B. 2000, J. Geophys. Res., 105,27447

Ferreira, S. E. S. 2002, The heliosperic transport of galactic cosmic rays and jovian electrons, Ph.D. Thesis

Ferreira, S. E. S., Potgieter, M. S., Burger, R. A., Heber, B., \& Fichtner, H. 2001a, J. Geophys. Res., 106, 24979

Ferreira, S. E. S., Potgieter, M. S., Burger, R. A., et al. 2001b, J. Geophys. Res., 106, 29313

Fichtner, H., Sreenivasan, S. R., \& Fahr, H. J. 1996, A\&A, 308, 248

Fisk, L. A. 1996, J. Geophys. Res., 101, 15547

Henize, V. K., Potgieter, M. S., \& Ferreira, S. E. S. 2003, Modeling a few-MeV Jovian and Galactic Electron Spectra in the Inner Heliosphere, 28th International Cosmic Ray Conference, 3819

Kanekal, S. G., Baker, D. N., Blake, J. B., et al. 2003, J. Geophys. Lett., 30, 1795

Kissmann, R. 2002, Modellierung des zeitabhaengigen Transports energetischer Elektronen in der Heliosphaere, Diploma-thesis, Ruhr-Universitaet-Bochum

Kissmann, R., Fichtner, H., \& Ferreira, S. E. S. 2004, A\&A, 419, 357

Langner, U. W., de Jager, O. C., \& Potgieter, M. S. 2001, Adv. Sp. Res., 27, 517

McComas, D. J., Elliott, H. A., \& Schwadron, N. A. 2003, J. Geophys Res., 30, 1517

Moeketsi, D. M., Potgieter, M. S., Ferreira, S. E. S., et al. 2005, Adv. Sp. Res., 35, 597

Morioka, A., Tsuchiya, F., \& Misawa, H. 1999, Earth Planets Space, 51,987

Parker, E. 1965, Planetary and Space Sci., 13, 9 